\title{
Densidad, abundancia y estructura poblacional del caracol blanco Strombus costatus en el Caribe Mexicano
}

Density, abundance and population structure of the milk conch Strombus costatus of the Mexican Caribbean

\author{
Virginia Balán-Dzul ${ }^{1}$ y Alberto de Jesús-Navarrete ${ }^{2}$
}

\begin{abstract}
${ }^{1}$ Instituto Tecnológico de Chetumal, Licenciatura en Biología, Av. Insurgentes 330, 77013 Chetumal, Quintana Roo, México ${ }^{2}$ Departamento de Manejo y Aprovechamiento de Recursos Acuáticos, El Colegio de la Frontera Sur, Unidad Chetumal, Av. Centenario Km 5.5, Apartado Postal 424, 77014 Chetumal, Quintana Roo, México. anavarre@ecosur.mx
\end{abstract}

\begin{abstract}
The milk conch Strombus costatus has rarely been studied at population and fisheries level in the Caribbean and Gulf of Mexico. To determine density and population structure of S. costatus at Banco Chinchorro, samplings was conducted using the method of distance during two seasons: summer (October 2008 and J uly 2009) and winter (November 2008 and February 2009), in order to evaluate the degree of aggregation we use the ratio variance/mean. With the size frequencies, growth parameters were obtained using FISAT II. 157 conchs were recorded, which showed an aggregate distribution. The highest density occurred in the winter season; 172 ind há-1 in the southern zone in November 2008, whereas in the central zone density was 77 ind há-1 in February 2009 and finally 51 ind há-1 in the northern zone in February 2009. The size structure varied between $80-165 \mathrm{~mm}$ siphonal length. The highest relative frequency value (FR) was detected in the south ranging between 111-115 mm LS. The infinite maximum length was $L_{\infty}=193.5$, and a growth constant $K=0.38$ with $t_{0}=-0.51$ was calculated. The rate of natural mortality was $M=1.062$. The reported densities are higher than those reported in other Caribbean areas; however, the population of S. costatus could not support commercial exploitation at Banco Chinchorro. Densities of S. costatus have not changed over time, so milk conch is not displacing queen conch, which is subject to fisheries.
\end{abstract}

Key words: Distance sampling, growth, Strombidae, natural mortality, population changes

Resumen. - El caracol blanco Strombus costatus se ha estudiado poco en el Caribe y el Golfo de México, tanto a nivel poblacional como pesquero. Para determinar la densidad y estructura poblacional de S. costatus en Banco Chinchorro, se realizaron muestreos con el método de la distancia, en dos temporadas del año: verano (octubre 2008 y julio 2009) e invierno (noviembre 2008 y febrero 2009), para el grado de agregación se usó la relación varianza/ media. Con las frecuencias de longitudes, se obtuvieron los parámetros de crecimiento, empleando FISAT II. Se registraron 157 caracoles, que mostraron una distribución agregada. La mayor densidad se concentró en la temporada de invierno, 172 ind há-1 en la zona sur en noviembre 2008, 77 ind há-1 en la zona centro en febrero de 2009 y 51 ind há-1 en la zona norte en febrero 2009. La estructura de tallas fluctuó entre $80-165 \mathrm{~mm}$ de longitud sifonal. El mayor valor de frecuencia relativa (FR) estuvo en la zona sur entre 111 a $115 \mathrm{~mm}$ de LS. La longitud máxima infinita fue de $L_{\infty}=193,5$, la constante de crecimiento $K=0,38$, con una $t_{0}=-0,51$. La tasa de mortalidad natural fue $M=1,062$. Las densidades encontradas son muy superiores a las reportadas en otras áreas del Caribe, no obstante, la población de S. costatus no podría tolerar una explotación comercial en Banco Chinchorro. Las densidades de S. costatus no han variado con el tiempo, por lo que el caracol blanco no está desplazando al caracol rosa que está suj eto a presión pesquera.

Palabras clave: Muestreo a distancia, crecimiento, Strombidae, cambios poblacionales, mortalidad natural

\section{INTRODUCCIÓN}

En la Península de Yucatán, la pesquería de gasterópodos es considerada multiespecífica, en Campeche y Yucatán, dentro de la familia Strombidae, se capturan el caracol blanco (Strombus costatus Gmelin, 1791) y el caracol peleador (Strombus pugilis Linnaeus, 1758) además de otras especies de las Familias: Buccinidae (Busycon sp.) y Turbinellidae (Xancus angulata). En Quintana Roo, las capturas se enfocan principalmente al caracol rosa Strombus gigas Linnaeus, 1758 y las otras especies se capturan en menor escala (De Jesús-Navarrete 2001, 2002). 
En Quintana Roo, el caracol reina o rosado, Strombus gigas, constituye la principal pesquería de moluscos, sin embargo, desde 1985 sus poblaciones han experimentado una disminución drástica, lo que ha propiciado que las cuotas de captura sean cada vez menores para las cooperativas pesqueras. Además, se observa un daño severo a la biomasa capturable debido a una pesca ilegal excesiva (De JesúsNavarrete et al. 2003). Una fuente alternativa para aliviar esta presión, y que los mismos pescadores han sugerido, podría ser la explotación de otras especies, entre ellas el caracol blanco Strombus costatus, que se captura en Yucatán, y ampliamente distribuido en el Caribe.

De las investigaciones que existen actualmente, Brownell (1977) en Trinidad y Tobago, estudió la distribución del género Strombus, incluyendo al caracol blanco, Appeldoorn (1985) en Puerto Rico, estudió el crecimiento, mortalidad y dispersión bajo condiciones de laboratorio, mientras que Berg et al. 1992 investigó en las Bermudas la abundancia y distribución de S. costatus. En México, Aldana et al. (1989, 2001), y Aldana \& Patiño (1998) estudiaron el efecto de la temperatura y alimentación en el crecimiento de larvas, así como las dietas usadas en el larvicultivo de varias especies de estrómbidos, incluido el caracol blanco. Pérez \& Aldana (2000) analizaron la distribución, abundancia y morfología de varias especies de caracoles, incluyendo $S$. costatus en la Península de Yucatán; De Jesús-Navarrete (2002) estudió la abundancia y distribución de larvas y adultos de $S$. costatus en Banco Chinchorro. Tewfik \& Guzmán (2003) determinaron la estructura poblacional de $S$. gigas y $S$. costatus en Bocas del Toro, Panamá. A pesar de esta información generada, aún se requieren investigaciones sobre el caracol blanco que contribuya al manejo de la especie y poder así determinar los efectos de la pesca sobre las poblaciones y predecir su explotación

Cuando se estima el estado de una población, es indispensable una determinación precisa de la densidad y la abundancia para evaluar la biomasa disponible. El método de la distancia (DISTANCE®), ha mostrado ser confiable y además permite estimar el tamaño de una población (Buckland et al. 2001). Este método se ha aplicado extensivamente en estudios de ecología terrestre, principalmente con aves y mamíferos (Buckland et al. 2001). Sin embargo, el método no se ha usado de manera amplia para evaluar poblaciones marinas. En el Mediterráneo, Katsanevakis $(2006,2007)$ lo empleó para estudiar la distribución y abundancia del bivalvo Pinna nobilis. El método se ha utilizado en el estudio de peces asociados al arrecife de coral (Letourneur et al. 1998), evaluación de poblaciones en peces demersales (Ensign et al. 1995) y en peces de lagos de agua dulce (Pink et al. 2007), pero no se tienen reportes previos de su aplicación en gasterópodos marinos. El método de distancia se basa en un transecto en línea y para cada organismo se registra la distancia perpendicular al transecto. Con esa información, se ajusta una función de detección, la cual se usará para estimar la proporción de organismos en el área de estudio de acuerdo al programa computacional DISTANCE® (Buckland et al. 2001). En el medio acuático, el método ha mostrado ser más eficiente que el transecto en banda, especialmente en poblaciones donde la detección de los organismos es difícil (Katsenevakis 2007) y el transecto circular usado para evaluar densidad de caracoles (Pérez \& Aldana 2000). Por otro lado, una de las consecuencias de la explotación excesiva de los recursos pesqueros es la disminución de la densidad de la población y un cambio en la talla promedio de los organismos. También sucede que cuando las poblaciones comparten hábitat, como es el caso de los estrómbidos, la especie que no está sujeta a explotación podría aprovechar la disminución de la otra especie, cambiando tanto su densidad como su talla promedio en el tiempo (Bene \& Tewfic 2003). Por lo tanto, y considerando la importancia biológica y económica de estos caracoles, y la necesidad de evaluar el estado de la población del caracol blanco, los objetivos de este trabajo fueron: determinar la densidad, abundancia y estructura poblacional de $S$. costatus, con el método de la distancia (DISTANCE ${ }^{\circledR}$ ) para definir si es posible una explotación comercial y comprobar si la densidad y la talla media de la población de caracol blanco se ha incrementado en el tiempo.

\section{Materiales y MÉtodos}

La reserva de la biosfera Banco Chinchorro (1847'18,23” $\mathrm{N}$ y $87^{\circ} 14^{\prime} 87,27^{\prime \prime}$ O) es un complejo arrecifal tipo plataforma emergente, localizado frente a la costa sureste de Quintana Roo, entre las poblaciones costeras de Río Indio y Xcalak. Se encuentra separado casi $30 \mathrm{~km}$ de la costa por un gran canal con profundidades que llegan a los 500 m (Jordán \& Martín 1987) (Fig. 1).

Los muestreos fueron trimestrales, abarcando dos temporadas del año: verano (octubre de 2008 y julio de 2009) e invierno (noviembre de 2008 y febrero de 2009). Se realizaron en tres zonas de distribución de Strombus costatus, las cuales fueron establecidas de acuerdo a la profundidad de la zona:

Zona Sur con un área de 15461 há (estación 1: $18^{\circ} 32^{\prime} 26,51^{\prime \prime} \mathrm{N}-87^{\circ} 18^{\prime} 57,13^{\prime \prime}$ O; estación 2: 18²3' $54,38^{\prime \prime} N-87^{\circ} 21^{\prime} 51,88^{\prime \prime} O$; estación 3: 18²9’52,93”, $\left.\mathrm{N}-87^{\circ} 25^{\prime} 54,11^{\prime \prime} \mathrm{O}\right)$. 


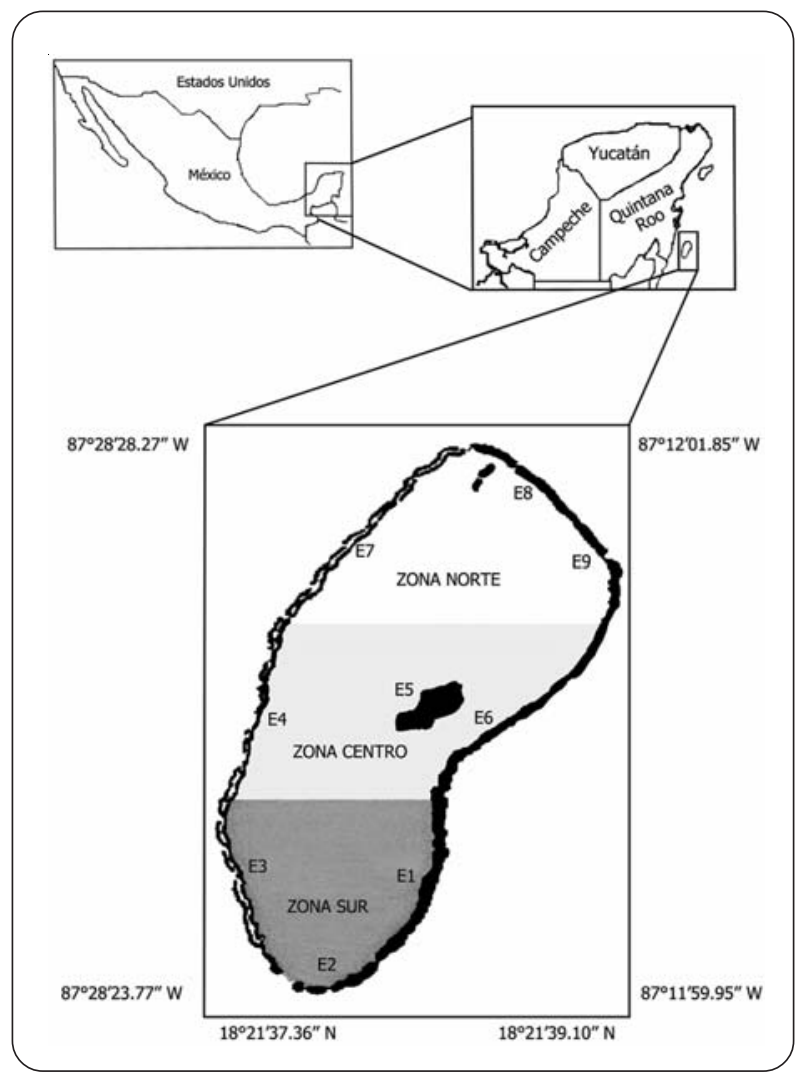

Figura 1. Ubicación del área de estudio, zonas y estaciones de muestreo (E1-E9) / Location of the study area, zone and sampling stations (E1- E9)

Zona Centro con área de 21110 há (estación 4:

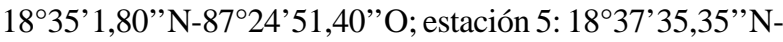
87²1'8,94”'O; estación 6: 18³4’15,34”N-87¹6’57,03”O).

Zona Norte con área de 17244 há (estación 7:

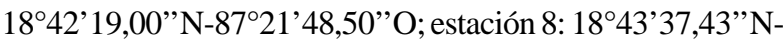
87¹5’38,48”O; estación 9: 18³6’15,21”N-87¹5’19,30”O).

En cada una de las nueve estaciones de la laguna arrecifal, se realizaron tres transectos de $100 \mathrm{~m}$ cada uno. En esas mismas áreas previamente se evaluó la densidad de $S$. costatus (De Jesús-Navarrete 2002).

Para determinar el tipo de distribución de los caracoles, se utilizó la relación varianza/media (VMR): (distribución aleatoria: VMR $=1,0$, distribución agregada: $\mathrm{VMR}>1$, distribución uniforme: VMR $\leq 1$ ). Posteriormente, se utilizó la prueba $\chi^{2}$ para determinar diferencias entre los coeficientes (Triola 2000).

Para determinar la densidad de los caracoles, se utilizó la metodología de toma de muestras a distancia: DISTANCE ${ }^{\circledR}$, los datos de la densidad se procesaron utilizando la función riesgo, con una serie polinomial, que fue la que mejor se ajustó en el cálculo de la densidad. (Buckland et al. 2001). Mediante buceo libre se realizó el muestreo y en cada estación se establecieron tres transectos lineales de $100 \mathrm{~m}$. Perpendicular al transecto se registró la distancia de los organismos hallados de los lados izquierdo y derecho, que después se llevaron a la embarcación, midiendo la longitud sifonal (LS) y el ancho del labio, con un vernier a una precisión de $1 \mathrm{~mm}$. Con los datos de densidad se realizó un ANDEVA multifactorial para determinar si existían diferencias de la densidad, en función de los siguientes factores: año, zona y temporada, utilizando el paquete estadístico STATGRAPHIC (2006).

Con los datos de frecuencia de tallas, se calcularon los parámetros poblacionales: $\mathrm{K}_{\text {y }} \mathrm{L}_{\infty}$ con la subrutina de Shepherd (1987). La mortalidad natural M, se evaluó con el método de Pauly (1980), empleando el paquete computacional FISAT II (Gayanilo et al. 2001).

\section{Resultados}

Se realizaron 27 transectos; 9 en cada zona que cubrieron un área 1,08 há (10800 m²). Se obtuvieron un total de 157 caracoles en todo el muestreo, siendo la mayoría juveniles, distribuidos en las tres zonas: sur, centro y norte.

De acuerdo a la relación varianza-media, se observó que la distribución fue agregada. La prueba chi-cuadrado $\left(\chi^{2}=\right.$ $5,99$, g.l. $=1, P=0,05)$, mostró que no hubo diferencias significativas y por lo tanto, la distribución para Strombus costatus en todos los sitios de muestreo fue agregada (Tabla 1).

La densidad encontrada mediante el empleo del método DISTANCE ${ }^{\circledR}$ para cada zona se indica en la Tabla 2, De acuerdo al análisis global de los resultados, el valor más alto se encontró en la zona sur con 172 ind há ${ }^{-1}$ en noviembre de 2008 (invierno), y las zonas centro y norte presentaron el valor más bajo de densidad con 5 ind há ${ }^{-1}$ en octubre (verano) y noviembre (invierno) de 2008, respectivamente. Sin embargo, en invierno se registraron las más altas densidades del muestreo en las tres zonas: en noviembre de 2008 zona sur 172 ind há ${ }^{-1}$, en febrero de 2009 las zonas: centro con 77 ind há ${ }^{-1}$ y la zona norte con 51 ind há ${ }^{-1}$. En cuanto a la estimación de densidad global por mes, el valor mayor de densidad de Strombus costatus fue en noviembre 2008; con una densidad de 66 ind há-1, y el mes con menor densidad fue julio (verano) de 2009 con una densidad de 34 ind há-1.

El análisis de varianza (ANDEVA) no mostró diferencias significativas de la densidad, entre zonas, temporadas, ni al comparar nuestros resultados con los realizados por de JesúsNavarrete (2002) en Banco Chinchorro (Tabla 3). 
Tabla 1. Relación varianza/media (R) para el caracol blanco Strombus costatus: media $(\mathrm{X})$ varianza $\left(\mathrm{S}^{2}\right)$, y tipo de distribución por zona de muestreo (zona sur, zona centro y zona norte) / Ratio variance/ mean ( $R$ ) for the milk conch Strombus costatus: mean $(\mathrm{X})$, variance $\left(\mathrm{S}^{2}\right)$, and type of distribution by sampling area (south, central and north zone)

\begin{tabular}{|c|c|c|c|c|c|}
\hline & Zonas & $\mathrm{x}$ & $S^{2}$ & $\begin{array}{c}\mathrm{R} \\
{\left[\mathrm{R}=\mathrm{S}^{2}(\mathrm{X})\right]}\end{array}$ & $\begin{array}{c}\text { Tipo } \\
\text { distribución }\end{array}$ \\
\hline \multirow[t]{6}{*}{ JULIO (2009) } & Zona sur & & & & \\
\hline & Est. $1,2,3$ & 1,22 & 2,44 & 2 & Agregada \\
\hline & Zona centro & & & & \\
\hline & Est. $4,5,6$ & 2 & 5 & 2,5 & Agregada \\
\hline & Zona norte & & & & \\
\hline & Est. 7,8,9 & 1,77 & 2,19 & 1,23 & Agregada \\
\hline \multirow[t]{6}{*}{ OCTUBRE (2008) } & Zona sur & & & & \\
\hline & Est. $1,2,3$ & 1,53 & 11,40 & 7,44 & Agregada \\
\hline & Zona centro & & & & \\
\hline & Est. $4,5,6$ & 1,33 & 2,33 & 1,75 & Agregada \\
\hline & Zona norte & & & & \\
\hline & Est. $7,8,9$ & 0,66 & 1,33 & 2 & Agregada \\
\hline \multirow[t]{6}{*}{ NOVIEMBRE (2008) } & Zona sur & & & & \\
\hline & Est. $1,2,3$ & 3,44 & 23,02 & 6,68 & Agregada \\
\hline & Zona centro & & & & \\
\hline & Est. $4,5,6$ & 1,33 & 5,33 & 4 & Agregada \\
\hline & Zona norte & & & & \\
\hline & Est. 7,8,9 & 0,33 & 0,33 & 1 & Agregada \\
\hline \multirow[t]{6}{*}{ FEBRERO (2009) } & Zona sur & & & & \\
\hline & Est. $1,2,3$ & 1,55 & 6,52 & 4,19 & Agregada \\
\hline & Zona centro & & & & \\
\hline & Est. $4,5,6$ & 2,44 & 13,27 & 5,43 & Agregada \\
\hline & Zona norte & & & & \\
\hline & Est. $7,8,9$ & 1,44 & 3,27 & 2,26 & Agregada \\
\hline
\end{tabular}

Tabla 2. Densidades (ind há ${ }^{-1}$ ) de Strombus costatus, en Banco Chinchorro / Densities (ind há-1) of Strombus costatus, at Banco Chinchorro

\begin{tabular}{|c|c|c|c|c|c|c|c|c|}
\hline \multirow[t]{2}{*}{ Temporada } & \multicolumn{2}{|c|}{ Zona Sur } & \multicolumn{2}{|c|}{ Zona Centro } & \multicolumn{2}{|c|}{ Zona Norte } & \multicolumn{2}{|c|}{ Global } \\
\hline & $\begin{array}{c}\mathrm{D} \\
\text { (ind há }{ }^{-1} \text { ) }\end{array}$ & $\% \mathrm{CV}$ & $\begin{array}{c}\mathrm{D} \\
\text { (ind há } \\
\text { ) }\end{array}$ & $\% \mathrm{CV}$ & $\begin{array}{c}\mathrm{D} \\
\text { (ind há }{ }^{-1} \text { ) }\end{array}$ & $\% \mathrm{CV}$ & $\begin{array}{c}\mathrm{D} \\
\text { (ind há } \\
\text { ) }\end{array}$ & $\% \mathrm{CV}$ \\
\hline \multicolumn{9}{|l|}{ Lluvias } \\
\hline Oct $/ 2008(\mathrm{n}=27)$ & 121 & 51,92 & 5 & 100 & 10 & 100 & 40 & 46,19 \\
\hline $\mathrm{Jul} / 2009(\mathrm{n}=44)$ & 23 & 42,64 & 32 & 47,89 & 47 & 44,90 & 34 & 27,67 \\
\hline \multicolumn{9}{|l|}{ Nortes } \\
\hline Nov/2008 $(n=36)$ & 172 & 46,44 & 39 & 115,47 & 5 & 100 & 66 & 43,63 \\
\hline $\mathrm{Feb} / 2009(\mathrm{n}=50)$ & 39 & 52,08 & 77 & 51,78 & 51 & 47,88 & 58 & 31,97 \\
\hline
\end{tabular}

D: densidad, \%CV: porcentaje de coeficiente de variación 
Tabla 3. Resultados de ANDEVA multifactorial de la densidad de S. costatus en Banco Chinchorro, Quintana Roo, México / Results of multifactorial ANOVA of S. costatus density at Banco Chinchorro, Quintana Roo, México

\begin{tabular}{lccccc}
\hline $\begin{array}{l}\text { Fuentes de } \\
\text { variación }\end{array}$ & $\begin{array}{c}\text { Suma de } \\
\text { cuadrados }\end{array}$ & $\begin{array}{c}\text { Grados de } \\
\text { libertad }\end{array}$ & $\begin{array}{c}\text { Cuadrado } \\
\text { medio }\end{array}$ & F-razón & $P$ \\
\hline Año & 0,0008 & 1 & 0,0008 & 4,36 & 0,075 \\
Temporada & 0,0002 & 1 & 0,0002 & 1,22 & 0,306 \\
Zona & 0,0005 & 2 & 0,0002 & 1,47 & 0,293 \\
Residual & 0,0013 & 7 & 0,0001 & & \\
Total & 0,0028 & 11 & & & \\
\hline
\end{tabular}

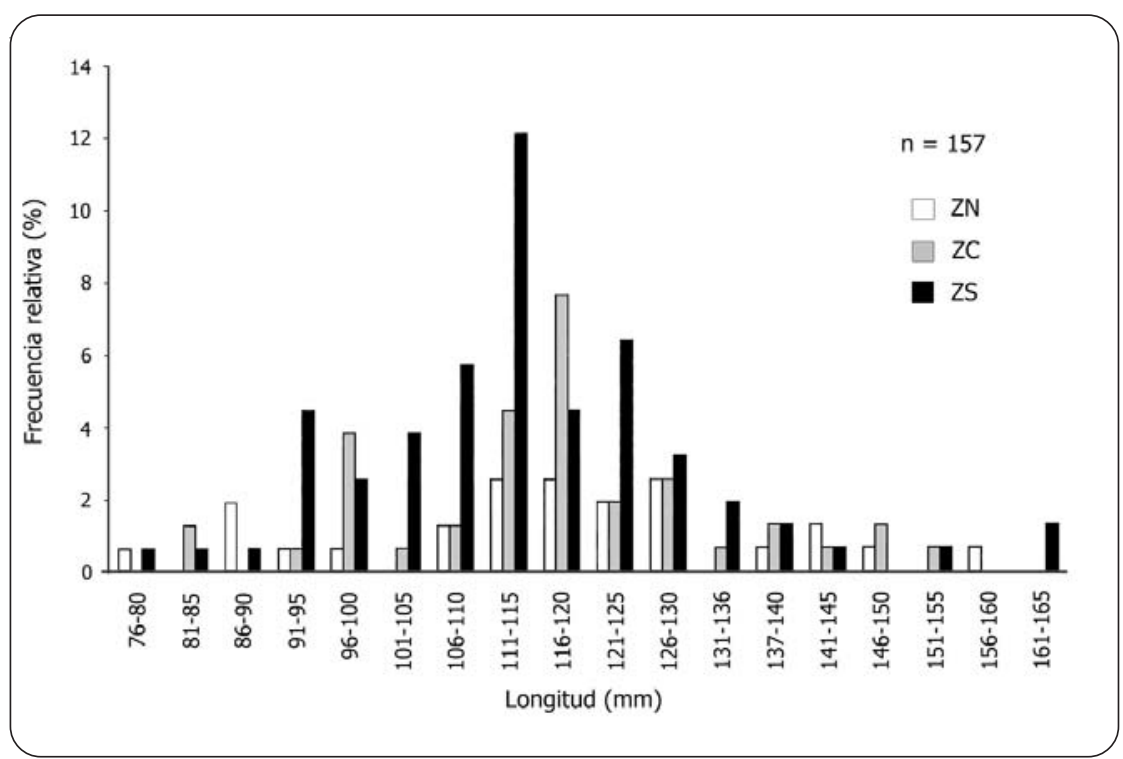

Figura 2. Distribución por zonas y tallas de Strombus costatus en Banco Chinchorro. $n$ : número total de $\mathrm{S}$. costatus registrados, $\mathrm{ZN}$ : zona norte, ZC: zona centro, ZS: zona sur / Distribution by zone and size of Strombus costatus in Banco Chinchorro. n: S. costatus total number registered, ZN: north zone, ZC: center zone, ZS: south zone
Las longitudes variaron entre 80 y $160 \mathrm{~mm}$ de longitud sifonal (Fig. 2). En la zona sur, los organismos presentaron tres picos de mayor frecuencia en longitudes de 106-110, 111-115 y 121-125 mm, respectivamente. En la zona centro, las longitudes variaron de 96-100, 111-115 y 116-120 mm. En la zona norte los principales picos de mayor frecuencia fueron de 111-115, 116-120 y 126-130 mm. La longitud promedio de $S$. costatus hallada en este estudio fue de 115 $\mathrm{mm}$, sin embargo en la zona sur fue de $113 \mathrm{~mm}$, en la zona centro fue $118 \mathrm{~mm}$ y zona norte de 116, siendo juveniles la mayor parte de la población muestreada.

Los parámetros de crecimiento encontrados fueron: $\mathrm{L}_{\infty}=$ $193,50, \mathrm{~K}=0,38, \mathrm{t}_{\mathrm{o}}=-0,517$, con un índice de bondad de ajuste de $R n=1,000$, la mortalidad natural calculada fue $\mathrm{M}$ = 1,062 al año.

\section{Discusión}

En general, dentro de los moluscos estrómbidos existe una tendencia a la agregación, que puede ocurrir en diferentes estadios, principalmente en la fase juvenil, o en la adulta, ésta última se relaciona con la reproducción (Randall 1964). En este trabajo, las 9 estaciones establecidas en Banco Chinchorro, las poblaciones de Strombus costatus presentaron una tendencia a la agregación, y coincide con lo que mencionaron Alcolado (1976) y Pérez \& Aldana (2000) quienes observaron el mismo comportamiento con S. gigas, que se asocia en grupos de tamaño y edad similar. Se observó que $S$. costatus coexistió con $S$. gigas en las tres zonas de muestreo, ya que ambas especies se encontraron en praderas de pastos marinos, y pedacería de coral, hecho que se ha descrito en otras áreas del Caribe, encontrándose 
también en camas de algas y arenales (Tewfik et al. 2003), donde posiblemente encuentren alimento suficiente para desarrollarse.

En Banco Chinchorro, se estimó para Strombus costatus, una densidad de 5 a 172 ind há-1 y una media de 51,75 ind há $^{-1}$, lo cual fue mayor a las reportadas en Bocas del Toro (1,27 ind há-1 $)$, en República Dominicana 50 y 200 ind há $^{-1}$ (Tewfik \& Guzmán 2003), en tanto que en Bermudas la densidad fue de 2,6 ind há-1 (Berg et al. 1992). Mientras que en estudios realizados en México, la densidad varió entre 0,06 y 50 ind há ${ }^{-1}$ en Yucatán. Para Banco Chinchorro, se registraron 50 ind há ${ }^{-1}$ en Cayo Lobos y 25 ind há ${ }^{-1}$ en Isla Che, 825 ind há ${ }^{-1}$ en Cayo Centro y 25 ind há ${ }^{-1}$ en Cayo Centro Oeste (Jesús-Navarrete 2002) (Tabla 4).

En Puerto Rico, Torres-Rosado (1987) encontró que los adultos de Strombus costatus fueron observados principalmente a profundidades de 10 a $28 \mathrm{~m}$ con mayor incidencia en los $22 \mathrm{~m}$. Probablemente, en Banco Chinchorro la mayor parte de la población del caracol blanco en edad reproductiva, se encuentre a profundidades mayores y sólo migren a zonas someras en época de reproducción. Este mismo hecho fue observado por Tewfik \& Guzmán (2003) e indicaron que el efecto de la baja densidad se refleja en la actividad reproductiva (copulación) de los organismos; si la densidad es menor a 56 ind há ${ }^{-1}$ por hectárea es muy probable que no ocurra. Por otro lado, la puesta de huevos con 48 ind há ${ }^{-1}$ es rara de observar (Stoner \& Ray-Culp 2000). En este trabajo sólo se observaron cinco parejas de $S$. costatus en actividad reproductiva, y no se encontraron masas de huevos, esto tal vez se deba a los meses en que se realizaron los muestreos, pero también puede ser un efecto de la baja densidad de organismos.

La distribución por longitudes de Strombus costatus en los diferentes sitios de muestreo, presentó condiciones similares a otros trabajos, Pérez \& Aldana (2000) reportaron para Yucatán una longitud sifonal máxima de $182 \mathrm{~mm}$ en Banco Chinchorro, de Jesús-Navarrete (2002) informó de una talla de $180 \mathrm{~mm}$ LS, mientras que para este estudio se encontro una longitud máxima de $160 \mathrm{~mm}$ LS. Las longitudes encontradas son similares a las obtenidas en muestreos previos (De Jesús-Navarrete, 2002) entre $30 \mathrm{~mm}$ a $180 \mathrm{~mm}$ con dos picos de mayor frecuencia uno en $81 \mathrm{~mm}$ y otro en $160 \mathrm{~mm}$, para este trabajo se presentaron dos picos uno en $91 \mathrm{~mm}$ y el otro en $130 \mathrm{~mm}$.

En cuanto a los parámetros de crecimiento para este estudio, se obtuvo una $\mathrm{L}_{\infty}=193,50, \mathrm{~K}=0,38 \mathrm{yt}_{\mathrm{o}}=-0,5137$. Estos parámetros son muy cercanos a los obtenidos por de Jesús-Navarrete (2002) donde la $\mathrm{L}_{\infty}=187,5, \mathrm{~K}=0,44 \mathrm{y} \mathrm{t}_{\mathrm{o}}=$
Tabla 4. Comparación de densidades medias de Strombus costatus en el Caribe / Comparison of mean densities of Strombus costatus in the Caribbean

\begin{tabular}{lrl}
\hline Lugar & Ind há $^{-1}$ & Referencia \\
\hline Bermuda & 2,60 & Berg (1992) \\
Yucatán, México & 9,60 & Pérez et al. (2000) \\
Banco Chinchorro, México & 139,10 & De Jesús-Navarrete (2002) \\
Bocas del Toro, Panamá & 1,27 & Tewfick \& Guzmán (2003) \\
Banco Chinchorro, México & 51,75 & Este estudio (2010) \\
\hline
\end{tabular}

-0,5362, lo que indicaría que los parámetros de crecimiento siempre concuerdan con las características de la población, observándose que Strombus costatus es una especie de crecimiento moderado y que puede llegar a tener como longitudes máximas, valores entre 187,5 mm y 193,50 mm. En contraste, Pérez \& Aldana (2000) obtuvieron una longitud máxima de 225 mm para el caracol blanco en Yucatán, a pesar que ahí si se explota al recurso.

La mortalidad total es el efecto expresado en la disminución del número de individuos de una cohorte a causa de varios factores y predecir lo que ocurre en una población (Tresierra et al. 1995), en este estudio la mortalidad natural fue mayor a la reportada por de JesúsNavarrete (2002), lo que nos hace pensar que los factores más importantes en la mortalidad, son los depredadores y fenómenos naturales que constantemente interactúan en la zona estudiada.

El método de la distancia fue una herramienta apropiada y eficaz para la evaluación de la densidad de caracol blanco, que permitió tener una estimación del número total de organismos en el área de trabajo y con menor esfuerzo de muestreo, considerando como una ventaja que la especie tiene una baja movilidad, y eso permite mayor precisión en las medidas. Es posible que un aumento en el número de transectos pueda incrementar el número de organismos capturados y mejorar los coeficientes de variación, con una mejor certeza de la evaluación, sin embargo, la variabilidad es un efecto que se ha observado también con otras especies de organismos, como los peces (Pink et al. 2007) y los bivalvos (Katsenavakis 2007). Con los resultados obtenidos, en términos de la distribución de tallas, y los parámetros de crecimiento de la especie, y con base en la densidad y número total de organismos, la población de Strombus costatus en Banco Chinchorro no podría sostener una pesquería de tipo comercial. Aparentemente no hay un incremento en la densidad de $S$. costatus en el área, ni un aumento en la talla 
media de la población, cuando se comparan con los datos de 1998, sin embargo, será necesario reunir otros datos sobre la especie, como estudios de desplazamiento y competencia, para determinar si hay un efecto de la disminución de una especie explotada (S. gigas), sobre otra que comparte el mismo hábitat y que no está sometida a presión pesquera.

\section{Agradecimientos}

A David González Sólis, Gerald Islebe y Juan Jacobo Schmitter por sus valiosos comentarios al manuscrito. A la Reserva de la Biosfera Banco Chinchorro, por las facilidades otorgadas en la realización de este trabajo. A Alejandro Medina Quej, José Juan Oliva Rivera y Aristeo Hernández Sánchez por su valioso apoyo y experiencia.

\section{LITERATURA CITADA}

Alcolado P. 1976. Crecimiento, variaciones morfológicas de la concha y algunos datos biológicos del cobo Strombus gigas L. (Mollusca, Mesogasteropoda). Serie Oceanológica, Academia de Ciencias, Cuba 34: 1-36.

Aldana D \& V Patiño. 1998. Overview of diets used in larviculture of three Caribbean conchs: queen conch, Strombus gigas, milk conch, Strombus costatus and fighting conch, Strombus pugilis. Aquaculture 167(3-4): 163-178.

Aldana D, E Baqueiro \& V Patiño. 2001. Descripción de las temperaturas usadas en el cultivo de larvas de Strombus spp. Proceedings of the 52nd Gulf and Caribbean Fisheries Institute 52: 327-339

Aldana D, A Lucas, T Brulé, F Salguero \& F Rendón. 1989. Effects of temperature, algal food, feeding rate and density on the larval growth of the milk conch (Strombus costatus) in Mexico. Aquaculture 76: 361-371.

Appeldoorn R. 1985. Growth, mortality and dispersion in juvenile, laboratory-reared conchs, Strombus gigas and $S$. costatus, released at an offshore site. Bulletin of Marine Science 37(3): 785-793.

Bene C \& A Tewfik. 2003. Biological evaluation of marine protected area: evidence of crowding effect on a protected population of queen conch in the Caribbean. P.S.Z.N. Marine Ecology 24(1): 45-58.

Berg C, F Couper, K Nisbet \& J Ward. 1992. Stock assessment of Queen Conch, Strombus gigas, and Harbour conch, S. costatus, in Bermuda. Gulf and Caribbean Fisheries Institute 41: 433-438.

Brownel W. 1977. Reproduction, laboratory culture and growth of Strombus gigas, Strombus costatus and Strombus pugilis, in Los Roques, Venezuela. Bulletin of Marine Science 27: 668-680.
Buckland S, T Anderson, D Burnham, K Laake, J Borchers \& D Thomas. 2001. Introduction to distance sampling: estimating abundance of biological populations, $440 \mathrm{pp}$. Oxford University Press, London.

De Jesús-Navarrete A. 2001. Crecimiento del caracol Strombus gigas (Gasteropoda: Strombidae) en cuatro ambientes de Quintana Roo, México. Revista de Biología Tropical 49(1): 83-89.

De Jesús-Navarrete A. 2002. Distribution and abundance of Strombus costatus (Gmelin, 1791) larvae and adults at the biosphere reserve: Banco Chinchorro, Quintana Roo, México. Journal of Shellfish Research 21(1): 227-231.

De Jesús-Navarrete A, A Medina \& J Oliva. 2003. Changes in the queen conch (Strombus gigas L.) population structure at Banco Chinchorro, Quintana Roo, México, 1990-1997. Bulletin of Marine Science 73(1): 219-229.

Ensign W, E Angermeier \& A Doldoff. 1995. Use of line transect method to estimate abundance of benthic stream fishes. Canadian Journal of Fisheries and Aquatic Sciences 52: 213-222.

Gayanilo F, P Sparre \& P Pauly. 2001. FAO-ICLARM stock assessment tools. Computerized information, 119 pp. Food and Agriculture Organization, Rome.

Jordán E \& E Martín. 1987. Chinchorro: Morphology and composition of a Caribbean. Atoll Research Bulletin 310: $1-20$.

Katsanevakis S. 2006. Population ecology of the endangered fan mussel Pinna nobilis in a marine lake. Endangered Species Research 1: 51-59.

Katsanevakis S. 2007. Density surface modeling with line transect sampling as a tool for abundance estimation of marine benthic species: the Pinna nobilis example in a marine lake. Marine Biology 152: 77-85.

Letourneur Y, M Kulbicki \& P Labrosse. 1998. Spatial structure of commercial reef fish communities along a terrestrial runoff in the northern lagoon of New Caledonia. Environmental Biology of Fishes 51: 141-159.

Pérez M \& D Aldana. 2000. Distribution, abundance and morphometrics of Strombus costatus, Turbinella angulata, Busycon contrarium and Pleuroploca gigantea (Mesogasteropoda: Strombidae, Turbinellidae, Neptuneidae and Fasciolaridae) in Yucatan, Mexico. Revista de Biología Tropical 48(1): 145-152.

Pink M, C Pratt \& G Fox. 2007. Use of underwater visual distance sampling for estimating habitat-specific population density. North American Journal of Fisheries Management 27: 246-255.

Randall J. 1964. Contributions to the biology of the queen conch S. gigas. Bulletin of Marine Science 14: 246-295. 
Stoner A \& M Ray-Culp. 2000. Evidence for Allee effects in an overharvested marine gastropod density-dependent mating and egg production. Marine Ecology Progress Series 202: 297-302.

Tewfik A \& H Guzmán. 2003. Shallow-water distribution and population characteristics of Strombus gigas and $S$. costatus (Gastropoda: Strombidae) in Bocas del Toro, Panamá. Journal of Shellfish Research 22(3): 789-794.

Tewfik A, H Guzmán \& G Jacome. 1998. Assessment of the queen conch. Strombus gigas (Gasteropoda, Strombidae) population in Cayos Cochinos, Honduras. Revista de Biología Tropical 46: 137-150.

Torres-Rosado Z. 1987. Distribution of two mesogastropods, the queen conch, Strombus gigas Linnaeus, and the milk conch, Strombus costatus Gmelin, in La Parguera, Lajas, Puerto Rico. M. S. Thesis, Universidad de Puerto Rico, Mayaguez, $37 \mathrm{pp}$.

Triola M. 2000. Estadística elemental, 824 pp. Addison Wesley Longman, México.

Recibido el 22 de abril de 2010 y aceptado el 19 de octubre de 2010 